\title{
Comparison of the porcine uterine smooth muscle contractility on days 12-14 of the estrous cycle and pregnancy
}

\author{
Włodzimierz Markiewicz ${ }^{1 *}$, Marek Bogacki ${ }^{2}$, Michał Blitek ${ }^{2}$ and Jerzy Jan Jaroszewski ${ }^{1}$
}

\begin{abstract}
Background: Uterine contractile activity is very important for many reproductive functions including embryo transport, implantation, gestation and parturition. Abnormal contractility leads to implantation failure, spontaneous miscarriage, preterm birth and many other disorders. The objective of the present study was to assess the effects of acetylcholine (ACh), noradrenaline (NA), oxytocin (OT) and prostaglandins $F_{2 a}\left(P_{G F}\right)$ and $E_{2}\left(P_{2} E_{2}\right)$ on the contraction of uterine strips collected from the horns of cyclic gilts (12-14 days of the estrous cycle-group I) and from pregnant (12-14 days after first insemination gilts in which one of the uterine horn was gravid (group lla) and the second one was non-gravid (group Illb). Uterine strips consisting of the endometrium with the myometrium and myometrium alone were examined.
\end{abstract}

Results: ACh increased the tension in all groups as compared to the pretreatment period, and the increase was the highest in group IIb; the amplitude decreased in all groups, and the frequency increased mainly in groups I and Ila. NA did not affect the tension in any group, but decreased the amplitude and frequency in group llb as compared to groups I and Ila. OT caused the highest increase in the tension in group IIb, a decrease in the amplitude and an increase in the frequency of contractions as compared to the pretreatment period. $\mathrm{PGF}_{2 a}$ induced the highest increase in the tension and amplitude in group $\mathrm{Ilb}$, with a decline in the frequency in this group. $\mathrm{PGE}_{2}$ increased the tension and frequency only in group $\mathrm{ll} \mathrm{b}$, and caused the greatest eduction in the amplitude in this group.

Conclusions: These results indicate that contractility of the porcine smooth muscle collected from uterine horns with embryos was different from those obtained from the uterine horns without embryos and the horns of cyclic gilts.

Keywords: Early pregnancy, Embryos, Gilts, Uterine contractility

\section{Background}

It is generally acknowledged that uterine contractility is regulated by complex interactions between many factors. Contractions determine the motor activity of the uterus which is particularly important during the migration of embryos and its implantation in the uterus. Contractions and relaxations of the porcine myometrium are controlled by the autonomic nerve system [1]. Moreover many endocrine and auto/paracrine/factors are also

\footnotetext{
*Correspondence: mark@uwm.edu.pl

${ }^{1}$ Department of Pharmacology and Toxicology, Faculty of Veterinary Medicine, University of Warmia and Mazury, Oczapowskiego Street 13, 10-718 Olsztyn, Poland

Full list of author information is available at the end of the article
}

involved in this regulation [2-4]. Uterine contractions in pigs can be stimulated by compounds such as acetylcholine (ACh) [5], oxytocin (OT) [6-8], prostaglandins $\mathrm{F}_{2 \alpha}\left(\mathrm{PGF}_{2 \alpha}\right)$ [7-9] and $\mathrm{E}_{2}\left(\mathrm{PGE}_{2}\right)[7,8,10]$, histamine [11], neuropeptide Y (NPY) [12], and endothelin [13]. On the other hand, the relaxation of the porcine myometrium may be caused by noradrenaline (NA), serotonin (5-HT) [14], and nitric oxide [15]. These factors influence smooth muscle contractility directly or indirectly by affecting the synthesis and release of other substances. It has been shown that both exogenous and endogenous ACh cause contraction of the myometrium through the activation of the muscarinic $\mathrm{M}_{3}$ receptor [5]. Other data have shown that NA causes an excitatory response, pre- 
dominantly via $\alpha_{2}$-adrenergic receptors, and stimulation of $\beta_{2}$-adrenergic receptors inhibits contractile activity of the porcine myometrium [1]. It is also generally accepted that OT stimulates uterine contractile activity via its receptors [6], which are present in the porcine uterus [16]. Oxytocin, acting through its receptors in the endometrium and myometrium, is involved in the control of $\mathrm{PGF}_{2 \alpha}$ and $\mathrm{PGE}_{2}$ secretion in pigs $[17,18]$. Prostaglandin $\mathrm{F}_{2 \alpha}$ contracts the uterine muscle indirectly by enhancing the responsiveness to OT followed by the prostaglandin $\mathrm{PGF}_{2}$ receptor $(\mathrm{FP})$ mediated regression of the corpora lutea (decrease in plasma progesterone levels) [2]. However, the direct action of $\mathrm{PGF}_{2 \alpha}$ on contractile $\left(\mathrm{FP}, \mathrm{EP}_{1}\right.$, $\mathrm{EP}_{3}$ ) and relaxatory (DP, IP, $\mathrm{EP}_{2}$ ) receptors in porcine uterine smooth muscle has also been described [2]. Similarly, $\mathrm{PGE}_{2}$ may cause contraction or relaxation through its impact on particular EP receptor subtypes [10].

In gilts, myometrial activity undergoes changes during the oestrous cycle $[2,9,10,14,19]$, at the time of mating and insemination [20,21], and during the course of pregnancy $[3,22]$. The early pregnancy in the pig is divided into three periods: post-conception (days 1-10 of pregnancy), the maternal recognition of pregnancy (days 11-13) and implantation (days 14-19) [23]. Thus, the period between the 12th and 14th days of the pregnancy is crucial for the successful implantation. The effects of various substances on motor activity of nonpregnant and pregnant porcine uteri have been studied, however, a comparison of the contractile activity between 12-14 days of the estrous cycle and pregnancy has not yet been made, especially in regard to the presence or not of embryos in the uterine horns. Therefore, the aim of our study was to examine the effects of ACh, $\mathrm{OT}, \mathrm{PGF}_{2 \alpha}$, and $\mathrm{PGE}_{2}$ on the contraction of uterine strips collected from the horns of cyclic gilts and from the horns with and without embryos of early pregnant pigs. Selection of the substances was made on their importance in the regulation of reproductive processes and the endogenous activity in the reproductive tract. The receptor mechanisms of these substances are also used in the case of drugs that interfere with the processes of uterine contractility. Until now, the differences in the impact of tested substances within the a period that is a crucial for a successful implantation compared to the analogous period of the estrous cycle are unknown. Therefore, we hypothesize that the uterine contractile response to stimuli will be affected by the presence of embryos within the uterus.

\section{Methods}

\section{Animals}

Prepubertal crossbred gilts $(\mathrm{n}=10)$ with a body weight of $106 \pm 4.8 \mathrm{~kg}$ and approximately 7 months of age were used. The gilts were subjected to surgical procedure under general anaesthesia. The animals were premedicated with azaperone $(2 \mathrm{mg} / \mathrm{kg}$ bw i.m.; Stresnil, Janssen Animal Health, Belgium) and ketamine $(12 \mathrm{mg} / \mathrm{kg}$ bw i.m.; VetaKetam, Vet-Agro, Poland), and anaesthetized with thiopental (20-30 mg/kg bw i.v.; Thiopental, Sandoz, GMBH, Austria). In five gilts one of the uterine horns was separated according to a surgically-generated model described previously by [24]. Briefly, the uterus was presented by a midventral opening of the caudal part of the abdomen. Thereafter, one horn was cut transversely and the ends were closed by a suture. This way the uterus consisted of one whole uterine horn and a part of the second horn, both connected with the uterine corpus. The remaining part of the second horn, connected with the contiguous ovary, was surgically detached from the uterine corpus. Ten days after surgery gilts were treated hormonally by an intramuscular injection of 750 I.U. of eCG (Folligon, Intervet, Poland) and 500 I.U. of hCG (Chorulon, Intervet) given $72 \mathrm{~h}$ later. Subsequently, $24 \mathrm{~h}$ after the hCG treatment the gilts were inseminated twice at $12 \mathrm{~h}$ intervals. Only gilts with the symptoms of heat were used for the next stages of procedures. Heat were checked by observation of symptoms: sticky discharge from vulva, clitoris red and protruding and the standing behaviour after applying pressure on the back and flanks of the gilt. The remaining five gilts were treated hormonally in the same way but they were not inseminated. On days 12-14 after insemination or for non-bred animals the gilts were slaughtered. To confirm pregnancy, the uterine horns were flushed with $10 \mathrm{ml}$ PBS to determine the presence of embryos in uterine flushings [24]. The exact number of embryos was not possible to count because of their defragmentation caused by flushing. All procedures involving animals were conducted in accordance with the rules approved by the Local Ethics Commission of the University of Warmia and Mazury in Olsztyn.

\section{Preparation of the uterine strips and measurements of their contraction}

Fragments of the uterine horns, collected from the middle part of the horns, were transferred to ice, moved to the laboratory and immediately processed for examination of contractile activity. Uterine tissue was collected from the horns of cyclic gilts (group I) and the horns of pregnant gilts without (group IIa) and with embryos (group IIb). The contractile activity was examined according to the method described previously [4, 19]. Briefly, two kinds of the uterine strips $(3 \times 5 \mathrm{~mm})$ consisting of the endometrium with myometrium (ENDO/ MYO) and myometrium (MYO) alone were resected. The study involved these two types of strips because in 
previous studies we found that the presence of endometrium affected the contractile activity of myometrium [20]. From each examined horn of the uterus two ENDO/MYO and two MYO strips were selected. After resection the strips were washed in saline and mounted between two stainless steel hooks in $5 \mathrm{ml}$ of an organ bath (Schuler Organ bath type 809; Hugo Sachs Electronic, Germany) under conditions of resting tension of $5 \mathrm{mN}$. The strips were kept in the Krebs-Ringer solution of the following composition (mM/l): $\mathrm{NaCl}, 120.3 ; \mathrm{KCl}$, 5.9; $\mathrm{CaCl}_{2}, 2.5 ; \mathrm{MCl}_{2}, 1.2 ; \mathrm{NaHCO}_{3}, 15.5$; glucose, 11.5; $37{ }^{\circ} \mathrm{C}, \mathrm{pH}$ 7.4. The solution was maintained at $37^{\circ} \mathrm{C}$ and continuously saturated with a mixture of $95 \mathrm{O}_{2}$ and $5 \%$ $\mathrm{CO}_{2}$. Measurements of smooth muscle contraction were conducted using a force transducer (HSE F-30 type 372), and a bridge coupler type 570, while the graphic recording was made on a recorder (Hugo Sachs Elektronik) with HSE-ACAD W software.

\section{Schedule of contractile activity examination}

The recording was started after prior equilibration for at least $60 \mathrm{~min}$. Thereafter, the strips were incubated with ACh $\left(10^{-5}-10^{-4} \mathrm{M}\right.$; Sigma, St. Louis, MO, USA), NA $\left(10^{-7}-10^{-6} \mathrm{M}\right.$; Levonor Polfa, Poland), OT $\left(10^{-7}-10^{-6}\right.$ $\mathrm{M}$, Vet-Agro, Poland), $\mathrm{PGF}_{2 \alpha}\left(10^{-8}-10^{-7} \mathrm{M}\right.$; Sigma), and $\mathrm{PGE}_{2}\left(10^{-8}-10^{-7} \mathrm{M}\right.$; Sigma). The doses of the substances tested were based on previous studies $[9,10,19$, 22]. Contractile activity was measured for $10 \mathrm{~min}$ after the administration of each concentration of the examined substance. At the end of the examination of each substance tissue chambers were washed three times with $15 \mathrm{ml}$ of Krebs-Ringer solution at 10 min intervals. Finally, at the end of treatment with examined substances to determine the viability of tissues, ACh was repeatedly administered in the same doses as given before. Only those results for which the difference in response to the stimulation by $\mathrm{ACh}$ at the beginning and the end of the treatment were less than $20 \%$ were included into the statistical analysis.

\section{Statistical analysis}

Numerical values of the contractile activity (intensity, amplitude and frequency) of the strips before the application of the examined substances were calculated for $10 \mathrm{~min}$ and accepted as $100 \%$. The results calculated for 10-min periods after treatments were expressed as a percentage (mean $\pm \mathrm{SD}$ ) of the contraction intensity, amplitude and frequency before drug administration. The statistical significance of the differences was assessed by one-way analysis of variance ANOVA (Graphpad PRISM 3.1; Graphpad Software, San Diego, CA, USA), followed by Bonferroni's multiple comparison test. Differences at $P<0.05$ were considered statistically significant.

\section{Results}

The spontaneous contractile activity before ACh administration in all examined tissues is shown in Fig. 1. Analysis of this activity did not demonstrate statistically significant differences between groups. Therefore, in the further statistical analysis the changes in the contractile activity after administration of the tested substances were compared to the pretreatment period.

\section{Influence of ACh on uterine contractile activity}

The administration of ACh in both doses caused a significant $(P<0.001)$ increase in the tension in all groups as compared to the pretreatment period (Fig. 2A). The magnitude of the increase was greater $(P<0.01-P<0.001)$
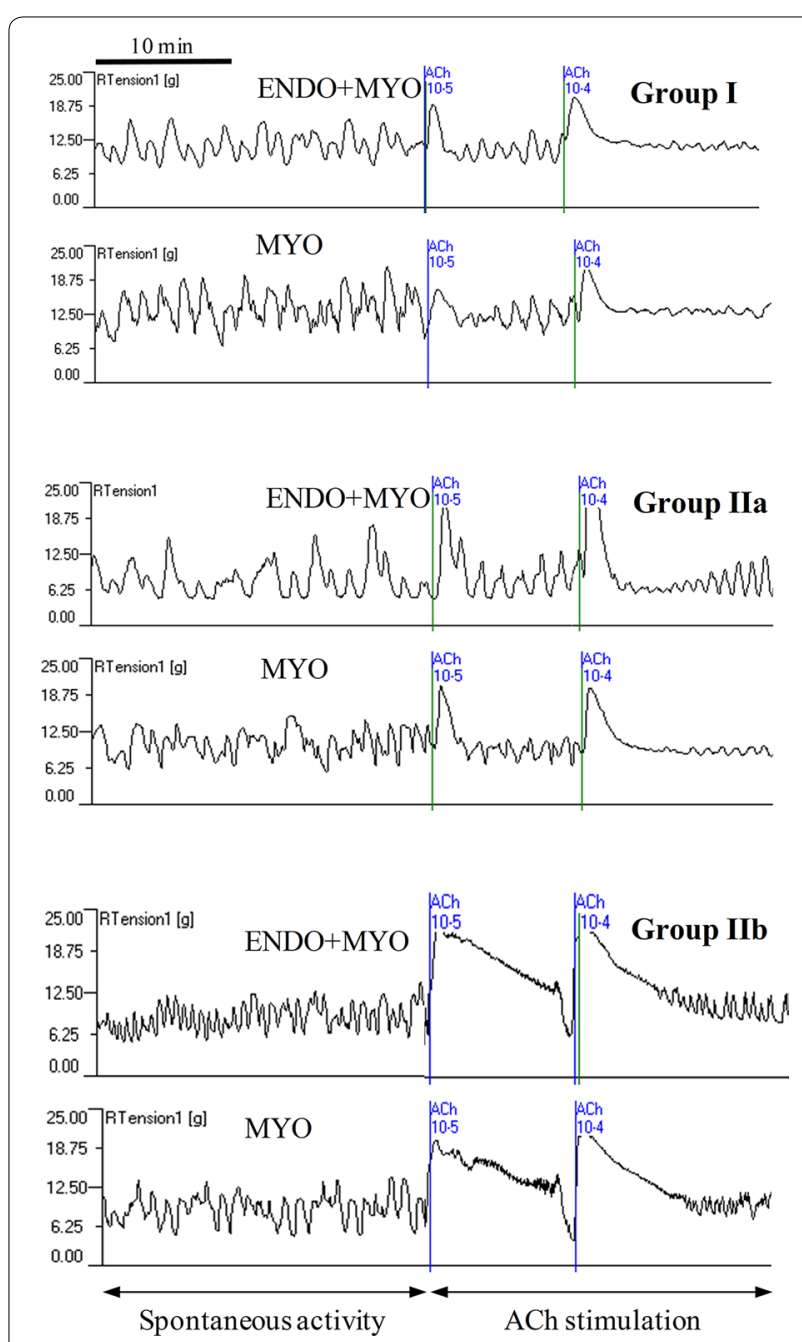

Fig. 1 A representative diagram showing spontaneous contractile activity and motor activity after acetylcholine (ACh) administration at doses $10^{-5}$ and $10^{-4} \mathrm{M}$ in the endometrium/myometrium (ENDO+MYO) and myometrium (MYO) strips collected on days 12-14 of the oestrous cycle (Group I) or after first insemination (groups without embryos-Group lla and with embryos-Group llb) 


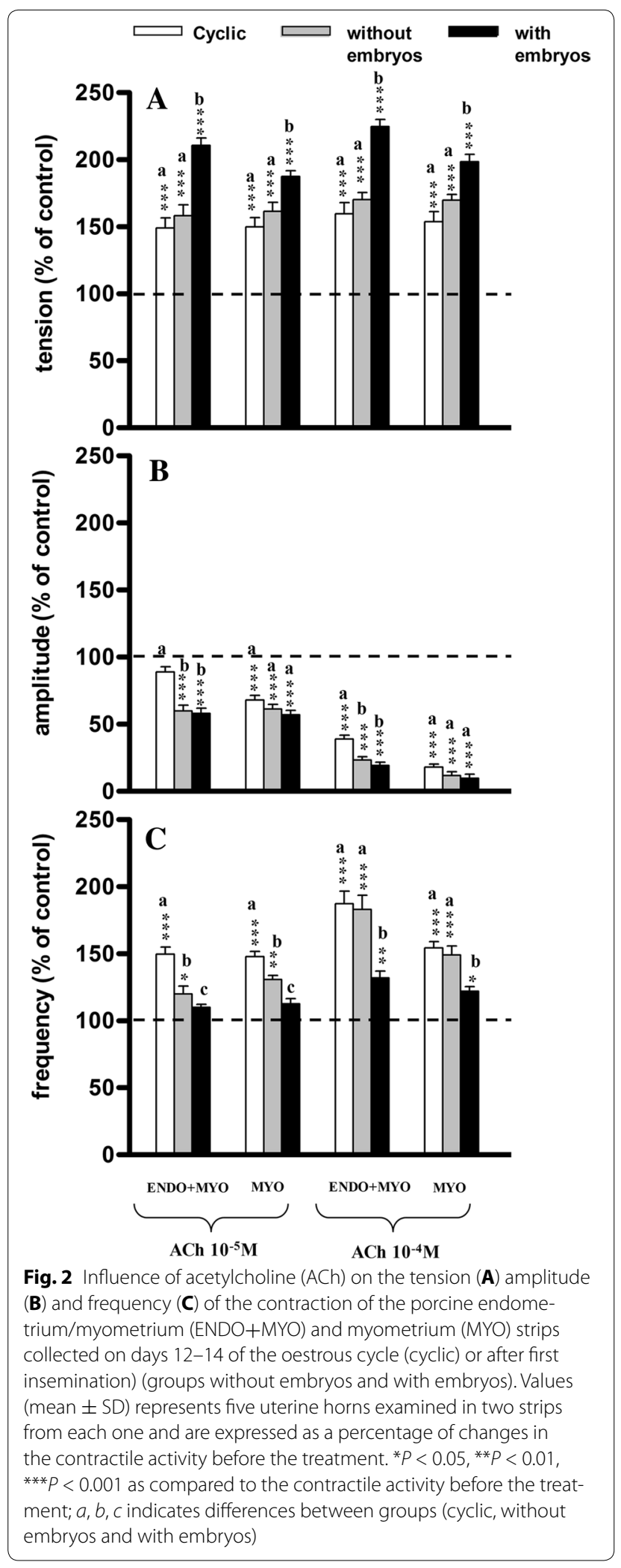

for strips from gravid horns than from non-gravid horns or cyclic gilt strips.

Administration of ACh caused a significant $(P<0.001)$ decrease in the amplitude in all groups (except ENDO/ MYO in group I at a concentration of $10^{-5} \mathrm{M}$ ) as compared to the pretreatment period (Fig. 2B). Moreover, significantly smaller changes in the amplitude of the ENDO/ MYO strips were determined in group I as compared to groups IIa and IIb $\left(P<0.001\right.$ after a dose of $10^{-5} \mathrm{M}$ and $P<0.05$ after a higher dose); such differences were not observed in the MYO strips.

The frequency of contractions in ENDO/MYO and MYO increased significantly $(P<0.05-P<0.001)$ after the application of $\mathrm{ACh}$ at both doses in groups $\mathrm{I}$ and IIa as compared to the pretreatment period (Fig. 2C). In group IIb a significant increase $(P<0.05-P<0.01)$ was observed only after the higher dose of ACh. After the lower dose of ACh the increase in frequency of contractions was significantly higher in the ENDO/MYO of group I as compared to groups IIa and IIb $(P<0.001)$, and in group IIa as compared to group IIb $(P<0.05)$; also in MYO strips a significantly greater increase was observed in group I as compared to groups IIa $(P<0.01)$ and IIb $(P<0.001)$, and in group IIa as compared to group IIb $(P<0.05)$. After the higher dose of ACh there were no differences between groups I and IIa, while a significantly $(P<0.05-P<0.001)$ lower increase in group IIb was observed in both kind of strips as compared to groups I and IIa.

\section{Influence of NA on uterine contractile activity}

Administration of NA at both doses insignificantly influenced tension as compared to the pretreatment period (Fig. 3A); such differences were also not observed between the experimental groups.

The amplitude of contractions was significantly $(P<0.001)$ decreased after both doses in the ENDO/ MYO and MYO in group IIb, and only after the smaller dose in groups I and IIa as compared to the pretreatment period (Fig. 3B). After NA administration in a dose of $10^{-7} \mathrm{M}$ significantly lower amplitude was observed in group IIb as compared to group I $(P<0.001)$, but this change was less evident as compared to group IIa $(P<0.05)$; significantly lower $(P<0.05-P<0.01)$ amplitude was also observed in group IIa as compared to group I in both kinds of the strips.

The frequency of contractions decreased significantly $(P<0.01-P<0.001)$ only in group IIb as compared to the pretreatment period (Fig. $3 \mathrm{C}$ ), as well as compared to groups I and IIa. 


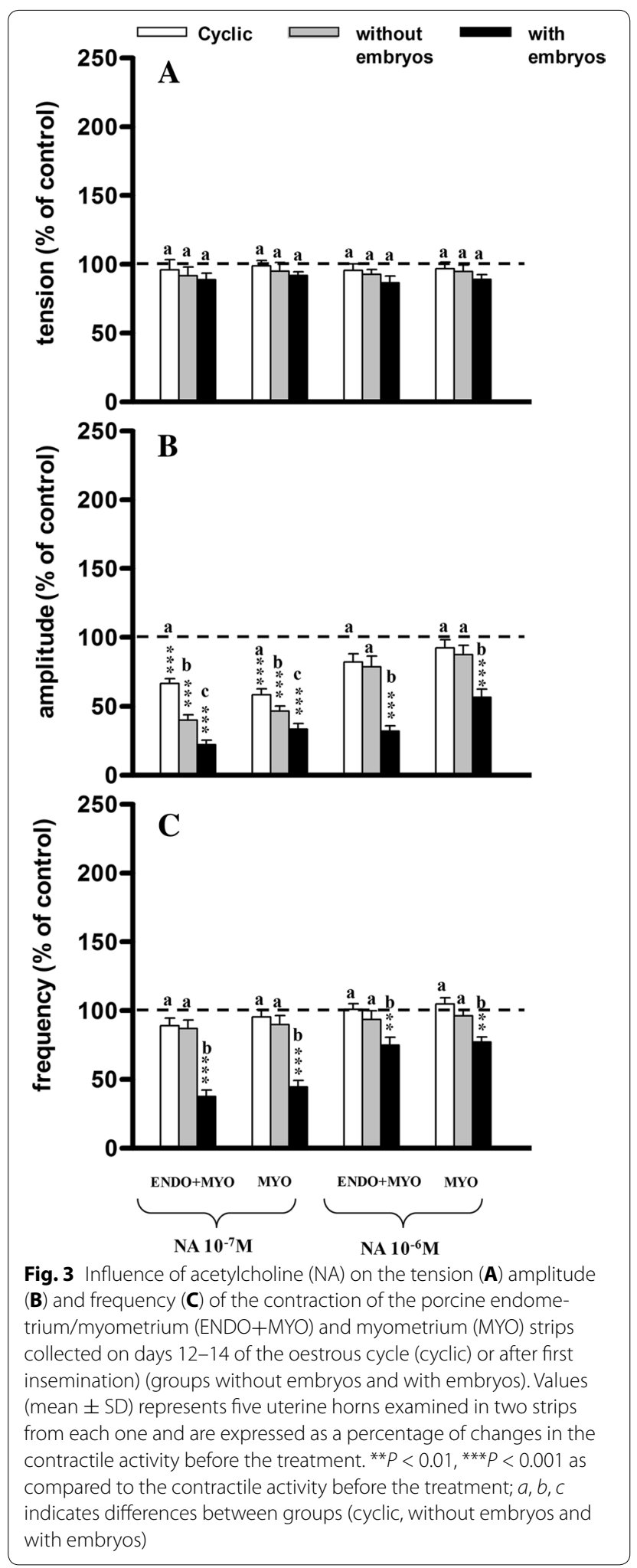

\section{Influence of OT on uterine contractile activity}

In the ENDO/MYO strips OT at both doses and in all groups caused a significant $(P<0.01-P<0.001)$ increase in tension as compared to the pretreatment period (Fig. 4A); a significant increase was also observed in the MYO strips in groups IIa and IIb. Insignificant differences $(P>0.05)$ were noticed in tension in both ENDO/ MYO and MYO strips between groups I and IIa, while significantly higher $(P<0.001)$ tension was found in group IIb as compared to groups I and IIa.

A significant $(P<0.05)$ increase in amplitude was observed after OT application at both doses in the ENDO/MYO and MYO strips in groups I and IIa, with a significant $(P<0.05)$ decrease in group IIb as compared to the pretreatment period (Fig. 4B). Significantly $(P<0.001)$ lower amplitude was also determined between group IIb as compared to groups I and IIa in both kinds of strip.

In ENDO/MYO and MYO strips the frequency of contractions decreased significantly in group I $(P<0.01-P<0.001)$ but increased in group IIb $(P<0.001)$, and was not changed in group Ila as compared to the pretreatment period (Fig. 4C). The frequency of contractions was significantly lower in group I as compared to groups IIa $(P<0.01)$ and IIb $(P<0.001)$, and in group IIa as compared to group IIb $(P<0.01-P<0.001)$.

\section{Influence of $\mathrm{PGF}_{2 \mathrm{a}}$ on uterine contractile activity}

In both kinds of strip a significant increase $(P<0.01-P<0.001)$ in tension after $\mathrm{PGF}_{2 \alpha}$ administration was observed only in group IIb as compared to the pretreatment period (Fig. 5A). There were no significant changes in tension between groups I and IIa, while significantly higher tension $(P<0.05-P<0.01)$ was observed in group IIb as compared to groups I and IIa.

$\mathrm{PGF}_{2 \alpha}$ significantly increased the amplitude in the ENDO/MYO strips in groups I $(P<0.05)$ and IIb $(P<0.001)$, and in the MYO strips in group IIb $(P<0.01)$ as compared to the pretreatment period (Fig. 5B). There were no significant changes in amplitude between groups I and IIa, while significantly higher $(P<0.01-P<0.001)$ amplitude was observed in group IIb as compared to groups I and IIa.

In the ENDO/MYO and MYO strips the frequency of contractions after $\mathrm{PGF}_{2 \alpha}$ administration decreased significantly $(P<0.001)$ in group IIb as compared to the pretreatment period (Fig. $5 \mathrm{C}$ ). There were no significant changes in the frequency of contractions between groups I and IIa, while significantly $(P<0.01-P<0.001)$ lower 

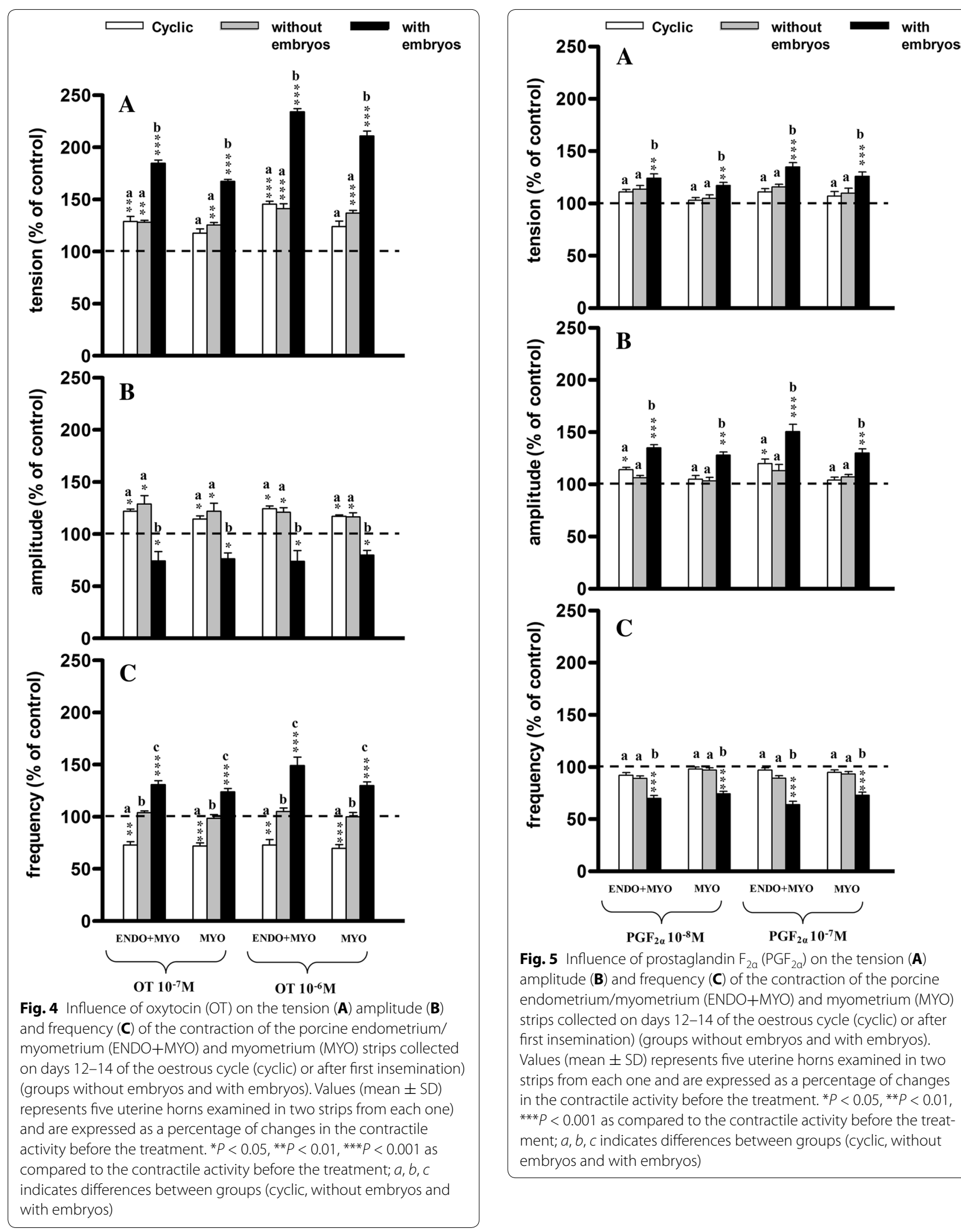

Fig. 5 Influence of prostaglandin $\mathrm{F}_{2 a}\left(\mathrm{PGF}_{2 a}\right)$ on the tension (A) amplitude $(\mathbf{B})$ and frequency $(\mathbf{C})$ of the contraction of the porcine endometrium/myometrium (ENDO+MYO) and myometrium (MYO) strips collected on days 12-14 of the oestrous cycle (cyclic) or after first insemination) (groups without embryos and with embryos). Values (mean \pm SD) represents five uterine horns examined in two strips from each one and are expressed as a percentage of changes in the contractile activity before the treatment. ${ }^{*} P<0.05,{ }^{*} P<0.01$, ${ }^{* * *} P<0.001$ as compared to the contractile activity before the treatment; $a, b, c$ indicates differences between groups (cyclic, without embryos and with embryos) 
frequency was observed in group IIb as compared to groups I and IIa.

\section{Influence of $\mathrm{PGE}_{\mathbf{2}}$ on uterine contractile activity}

At both doses $\mathrm{PGE}_{2}$ caused a significant increase $(P<0.001)$ in tension only in the ENDO/MYO strips from group IIb as compared to the pretreatment period (Fig. 6A), as well as compared to groups I and IIa $(P<0.01-P<0.001)$.

In the ENDO/MYO strips $\mathrm{PGE}_{2}$ at the smaller dose decreased the amplitude in group IIb $(P<0.001)$, whereas it decreased the amplitude at the higher dose in all groups $(P<0.01-P<0.001)$ as compared to the pretreatment period (Fig. 6B). Significantly lower amplitude was observed in the ENDO/MYO in group IIb as compared to group I $(P<0.01)$ and group IIa $(P<0.05)$.

The frequency of contractions did not change significantly in any of the analysed groups as compared to the pretreatment period (Fig. 6C). The frequency of contractions significantly $(P<0.05)$ increased in the ENDO/ MYO strips of group IIb as compared to groups I and IIa.

\section{Discussion}

The presented results demonstrate the influence of biologically active substances on smooth muscle contractility collected from cyclic gilts and uterine horns with or without embryos. The results indicate that in the uterus with embryos contractile activity is increased in comparison to the uterus with not embryos. These differences were clearer when compared to contractility in the uterus of cyclic gilts. The experiments in vitro of Pope et al. [25] showed that myometrial contractility increased concomitantly with embryo migration.

In our studies the ACh increased tension in the smooth muscle of the uterus, which was the highest in the strips of pregnant uterus, with a simultaneous decrease in amplitude. Moreover, the frequency of contractions in the strips of the uterus without embryos was higher as compared to the uterine horn with embryos. Our results are consistent with the results of other authors. Kim et al. [26] observed that after administration of carbachol, a muscarinic receptor agonist, the force of contraction of the uterus in pregnant rats was several times higher than in the non-pregnant uterus. Kitazawa et al. [5] found that both exogenous and endogenous ACh cause contraction of the uterine muscle in the pig by the activation of the muscarinic $\mathrm{M}_{3}$ receptor. Our results also indicated that in the uterine strips with embryos the highest increase in tension is combined with a higher decrease of amplitude

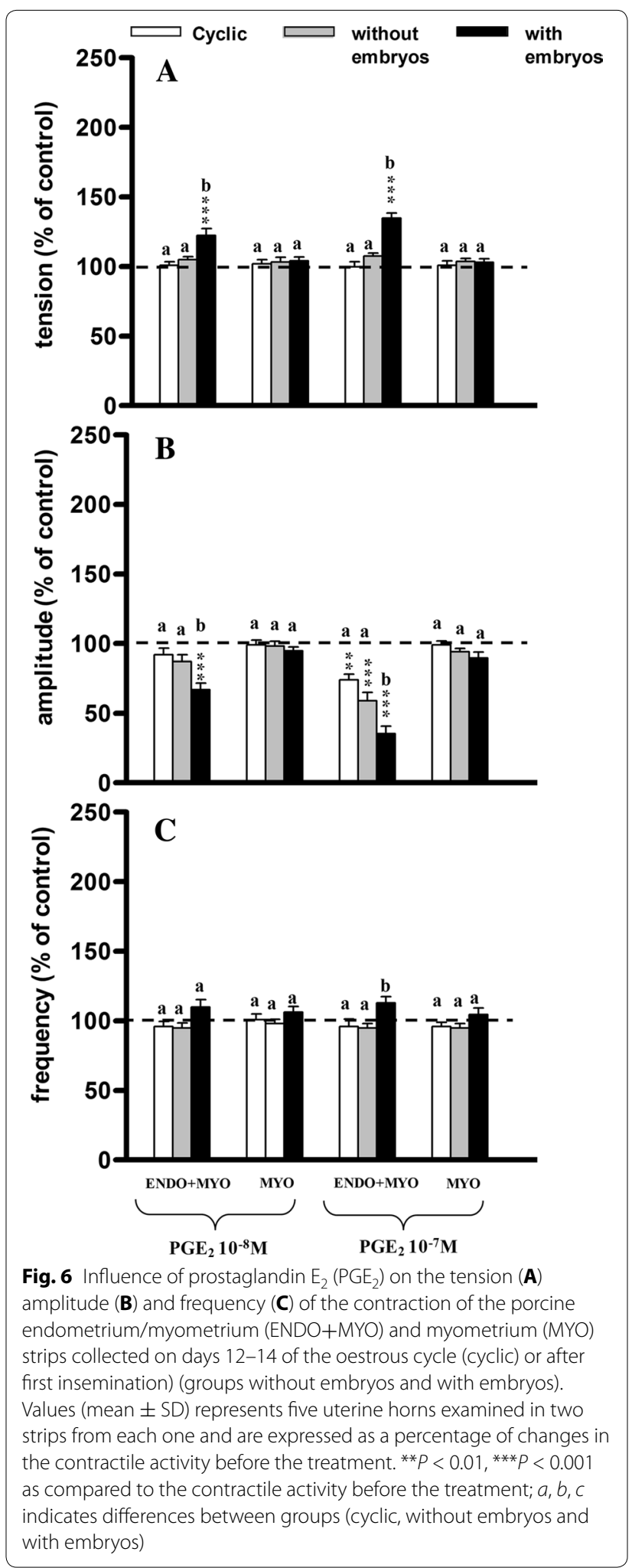


and a lower increase in the frequency of contractions as compared to the other groups examined. This data indicate that the high motor activity in the gravid horn can promote uniform distribution of the multiple embryos in the lumen of the pig uterus.

One of the regulatory mechanisms affecting uterine contractile activity is the stimulation of $\alpha$ - and $\beta$-adrenergic receptors by catecholamines such as NA. In our study, we observed that NA had no significant effect on muscle tension in any group during the 10-min observation period, although in some strips (in each group) a short-term increase in tension after its administration was seen. However, in comparison to the group I NA had a significant influence on the changes of amplitude and frequency of contractions in group IIb, which suggests that the presence of the embryo in the uterus affects the regulation of contractile activity. It is suggested that inhibition of uterine contractile activity is due to a prevalence of $\beta$-adrenergic over the $\alpha$-adrenergic receptors, and may be related to hormonal changes, particularly in the levels of ovarian steroid hormones, which affect the concentration and distribution of the population of muscarinic and adrenergic receptors [27-30]. Rexroad and Guthrie [31] suggested that a reduction in the number of $\alpha$-adrenergic receptors in the early days of pregnancy may be associated with the migration and implantation of embryos. In this way, the embryos may affect the uterus, e.g., causing changes in the expression of maternal factors. Furthermore, it was observed that embryos may physically affect the uterus by, e.g., changing the expression of genes responsible for the release of biologically active substances [32].

In our study the greatest increase in the tension and frequency of contractions after OT administration was observed in group IIb. Franczak and Bogacki [18] using the same pig model showed that embryonic products locally regulate the abundance of OT receptors mRNA in the porcine uterus. Moreover, it has been shown that the number of receptors for this peptide at 14-16 days of gestation, compared with days 14-16 of the cycle, is similar in MYO but much higher in ENDO [16]. The role of OT receptors in early pregnancy is not fully understood. It is assumed that the peptide, by interaction with its receptors, affects the secretion of prostaglandins, including $\mathrm{PGF}_{2 \alpha}$ and $\mathrm{PGE}_{2}$.

In our study, $\mathrm{PGF}_{2 \alpha}$ caused the largest increase in tension and amplitude in group IIb, with a decline in frequency in all groups. The weakest effect among all the tested substances was observed after $\mathrm{PGE}_{2}$ treatment. However, in the ENDO/MYO strips of group IIb it increased the tension and frequency and decreased the amplitude of contractions. In the reproductive system prostaglandin $\mathrm{F}_{2 \alpha}$ and $\mathrm{E}_{2}$ participates in the control of many process, including implantation of embryos and causing contractions of the uterus [33]. Dittrich et al. [34] demonstrated that human seminal plasma (consisting among others prostaglandins) has a direct effect on enhancing uterine contractility, which could facilitate sperm transport. It is suggested that prostaglandin secretion disorders during pregnancy are one of the causes of miscarriages and premature births. Currently, it is known that the myometrium secretes more $\mathrm{PGE}_{2}$ than $\mathrm{PGF}_{2} \alpha$ regardless of pregnancy status, and that the myometrium may be an additional important source of luteotrophic $\mathrm{PGE}_{2}$ action during early pregnancy [17]. It was also found that PGs may play a significant role in embryo implantation and decidualization of the endometrium [23].

Kitazawa et al. [3], studying the activity of smooth muscle of the uterus of pregnant pig, stated, similarly to our study, that $\mathrm{ACh}, \mathrm{OT}$ and $\mathrm{PGF}_{2 \alpha}$ increased contractility in muscle. However, in our study the tension and frequency of contractions were increased, while Kitazawa et al. [3] observed a reduction in the frequency of contractions. This difference may be due to the fact that in our study strips were taken at 12-14 days after first insemination, whereas Kitazawa et al. [3] used tissue from 25-60 days of pregnancy. It is accepted that pregnancy changes the expression of receptors, such as oxytocin receptor [35, 36] $\alpha_{2}$-adrenoceptor [37] and prostanoids receptors [38]. Therefore, the increase in the expression of contractile receptors in the uteri of pregnant pigs was suggested to be another mechanism for the pregnancy-associated increase in the contractile responses.

\section{Conclusions}

Summarizing, ACh, OT, $\mathrm{PGF}_{2 \alpha}$ and $\mathrm{PGE}_{2}$ significantly increased the tension and decreased the amplitude of contraction (except $\mathrm{PGF}_{2 \alpha}$ whose administration increased the amplitude) in gravid horns compared to non-gravid horns and cyclic gilts. The frequency of contractions was increased in the uterine horn with embryos after the administration of ACh and OT, but this parameter decreased after NA and $\mathrm{PGF}_{2 \alpha}$, and remained practically unchanged after the administration of $\mathrm{PGE}_{2}$. Surprisingly, the contractility of the uterine smooth muscle collected from gravid horns exhibited higher activity than that obtained from non-gravid horns and cyclic gilts. Perhaps this is connected with the time of implantation and uniform distribution of embryos. From the data of this study it seem possible to speculate that the presence of embryos may increase the release of some substances which, by auto and paracrine regulation, affect uterine contractile activity. 


\section{Authors' contributions}

MW conceived the study, and participated in its design, coordination and carried, and drafted the manuscript. MB took part in planning the experimental in vivo study and contributed to the set-up of the materials and methods. MB performed surgical procedures and collected materials for in vitro examinations. JJ J have been involved in revising it critically for important intellectual content and approval of the version to be published. All authors read and approved the final manuscript.

\section{Author details}

${ }^{1}$ Department of Pharmacology and Toxicology, Faculty of Veterinary Medicine, University of Warmia and Mazury, Oczapowskiego Street 13, 10-718 Olsztyn, Poland. ${ }^{2}$ Institute of Animal Reproduction and Food Research, Polish Academy of Sciences, Bydgoska Street 7, 10-243 Olsztyn, Poland.

\section{Acknowledgements}

The study was supported by the Grant No. 15.610.008-300 from the University of Warmia and Mazury in Olsztyn. Publication costs were covered by KNOW (Leading National Research Centre) Scientific Consortium "Healthy AnimalSafe Food", decision of Ministry of Science and Higher Education No. 05-1/ KNOW2/2015.

\section{Competing interests}

The authors declare that they have no competing interests.

Received: 12 December 2015 Accepted: 15 March 2016 Published online: 22 March 2016

\section{References}

1. Taneike T, Narita T, Kitazawa T, Bando S, Teraoka H, Ohga A. Binding and functional characterization of alpha-2 adrenoceptors in isolated swine myometrium. J Auton Pharmacol. 1995;15:93-105.

2. Cao J, Shayibuzhati M, Tajima T, Kitazawa T, Taneike T. In vitro pharmacological characterization of the prostanoid receptor population in the nonpregnant porcine myometrium. Eur J Pharmacol. 2002;442:115-23.

3. Kitazawa T, Hatakeyama H, Cao J, Taneike T. Pregnancy-associated changes in responsiveness of the porcine myometrium to bioactive substances. Eur J Pharmacol. 2003;469:135-44

4. Markiewicz W, Kamińska K, Bogacki M, Maślanka T, Jaroszewski JJ. Participation of analogues of lysophosphatidic acid (LPA): oleoyl-snglycero-3-phosphate (L-alpha-LPA) and 1-oleoyl-2-O-methyl-rac-glycerophosphothionate (OMPT) in uterine smooth muscle contractility of the pregnant pigs. Pol J Vet Sci. 2012;15:635-43.

5. Kitazawa T, Uchiyama F, Hirose K, Taneike T Characterization of the muscarinic receptor subtype that mediates the contractile response of acetylcholine in the swine myometrium. Eur J Pharmacol. 1999;367:325-34.

6. Kitazawa T, Kajiwara T, Kiuchi A, Hatakeyama H, Taneike T. Muscle layerand region-dependent distributions of oxytocin receptors in the porcine myometrium. Peptides. 2001;22:963-74.

7. Mueller A, Maltaris T, Siemer J, Binder H, Hoffmann I, Beckmann MW, et al. Uterine contractility in response to different prostaglandins: results from extracorporeally perfused non-pregnantswine uteri. Hum Reprod. 2006;21:2000-5.

8. Dittrich R, Mueller A, Oppelt PG, Hoffmann I, Beckmann MW, Maltaris T. Differences in muscarinic-receptor agonist-, oxytocin-, and prostaglandin induced uterine contractions. Fertil Steril. 2009;92:1694-700.

9. Kucharski J, Jaroszewski JJ, Jana B, Górska J, Kozłowska A, Markiewicz W. The influence of inflammatory process on prostaglandin F2alpha contractile activity in porcine uterus. J Anim Feed Sci. 2007;16:654-67.

10. Jana B, Jaroszewski JJ, Kucharski J, Koszykowska M, Górska J, Markiewicz W. Participation of prostaglandin E2 in contractile activity of inflamed porcine uterus. Acta Vet Brno. 2010;79:249-59.

11. Kitazawa T, Shishido H, Sato T, Taneike T. Histamine mediates the muscle layer-specific responses in the isolated swine myometrium. J Vet Pharmacol Ther. 1997;20:187-97.

12. Markiewicz W, Jaroszewski JJ, Bossowska A, Majewski M. NPY: its occurrence and relevance in the female reproductive system. Folia Histochem Cytobiol. 2003;41:183-92.
13. Isaka M, Takaoka K, Yamada Y, Abe Y, Kitazawa T, Taneike T. Characterization of functional endothelin receptors in the porcine myometrium. Peptides. 2000;21:543-51.

14. Kitazawa T, Nakagoshi $K$, Teraoka H, Taneike T. 5-HT(7) receptor and $\beta_{2}$ adrenoceptor share in the inhibition of porcine uterine contractility in a muscle layer-dependent manner. Eur J Pharmacol. 2001;433:187-97.

15. Buxton IL. Regulation of uterine function: a biochemical conundrum in the regulation of smooth muscle relaxation. Mol Pharmacol. 2004;65:1051-9.

16. Franczak A, Ciereszko R, Kotwica G. Oxytocin (OT) action in uterine tissues of cyclic and early pregnant gilts: OT receptors concentration, prostaglandin $\mathrm{F}_{2 a}$ secretion, and phosphoinositide hydrolysis. Anim Reprod Sci. 2005:88:325-39.

17. Franczak A, Kurowicka B, Oponowicz A, Petroff BK, Kotwica G. The effect of progesterone on oxytocin-stimulated intracellular mobilization of $\mathrm{Ca}^{2+}$ and prostaglandin $E_{2}$ and $F_{2 a}$ secretion from porcine myometrial cells. Prostaglandins Other Lipid Mediat. 2006;81:37-44.

18. Franczak A, Bogacki M. Local and systemic effects of embryos on uterine tissues during early pregnancy in pigs. J Reprod Dev. 2009;55:262-72.

19. Jana B, Jaroszewski JJ, Czarzasta J, Włodarczyk M, Markiewicz W. Synthesis of prostacyclin and its effect on the contractile activity of the inflamed porcine uterus. Theriogenology. 2013;79:470-85.

20. Langendijk P, Bouwman EG, Soede NM, Taverne MA, Kemp B. Myometrial activity around estrus in sows: spontaneous activity and effects of estrogens, cloptrostenol, seminal plasma and clenbuterol. Theriogenology. 2002:57:1563-77.

21. Langendijk P, Soede NM, Kemp B. Uterine activity, sperm transport, and the role of boar stimuli around insemination in sows. Theriogenology. 2005:63:500-13.

22. Kurowicka B, Franczak A, Oponowicz A, Kotwica G. In vitro contractile activity of porcine myometrium during luteolysis and early pregnancy: effect of oxytocin and progesterone. Reprod Biol. 2005;5:151-69.

23. Zięcik AJ, Wacławik MM, Kaczmarek A, Blitek B, Moza Jalali B, Andronowska A. Mechanisms for the establishment of pregnancy in the pig. Reprod Domest Anim. 2011;46:31-41.

24. Wasielak M, Głowacz M, Kamińska K, Wacławik A, Bogacki M. The influence of embryo presence on prostaglandins synthesis and prostaglandin E2 and F2alpha content in corpora lutea during periimplantation period in the pig. Mol Reprod Dev. 2008;75:1208-16.

25. Pope WF, Maurer RR, Stormshak F. Intrauterine migration of the porcine embryo-interaction of embryo, uterine flushings and indomethacin on myometrial function in vitro. J Anim Sci. 1982;55:1169-78.

26. Kim BK, Ozaki H, Hori M, Takahashi K, Karaki H. Increased contractility of rat uterine smooth muscle at the end of pregnancy. Comp Biochem Physiol. 1998;121:165-73.

27. Roberts JM, Insel PA, Goldfien RD, Goldfien A. a-adrenoceptors but not $\beta$-adrenoceptors increase in rabbit uterus with oestrogen. Nature. 1977;270:624-5.

28. Williams LT, Lefkowitz RJ. Regulation of rabbit myometrial receptors by estrogen and progesterone. J Clinic Invest. 1977;60:815-8.

29. Choppin A, Stepan GJ, Loury DN, Watson N, Eglen RM. Characterization of the muscarinic receptor in isolated uterus of sham operated and ovariectomized rats. Br J Pharmacol. 1999;127:1551-8.

30. Ontsouka EC, Reist M, Graber H, Blum JW, Steiner A, Hirsbrunner G. Expression of messenger RNA coding for 5-HT receptor, alpha and beta adrenoreceptor (subtypes) during oestrus and dioestrus in the bovine uterus. J Vet Med A Physiol Pathol Clin Med. 2004;51:385-93.

31. Rexroad CE Jr, Guthrie HD. Alpha-adrenergic receptors in myometrium of pregnant and nonpregnant pigs until day 19 postestrus. Biol Reprod. 1983:29:615-9.

32. Kennedy TG, Gillio-Meina C, Phang SH. Prostaglandins and the initiation of blastocyst implantation and decidualization. Reproduction. 2007;134:635-43.

33. Herschman HR. Prostaglandin synthase 2. Biochim Biophys Acta. 1996;1299:125-40.

34. Dittrich R, Henning J, Maltaris T, Hoffmann I, Oppelt PG, Cupisti S, et al. Extracorporeal perfusion of the swine utersu: effect of of human seminal plasma. Andrologia. 2012:44:543-9.

35. Fuchs AR, Fuchs F, Husslein P, Soloff MS, Fernstrom MJ. Oxytocin receptors and human parturition: a dual role for oxytocin in the initiation of labor. Science. 1982:215:1396-8. 
36. Fuchs AR, Fuchs F, Husslein P, Soloff MS. Oxytocin receptors in the human uterus during pregnancy and parturition. Am J Obstet Gynecol. 1984;150:734-41.

37. Arkinstall $\mathrm{S}$, Jones $\mathrm{CT}$. [3H]rauwolscine binding to myometrial $\mathrm{a}_{2}$ adrenoceptors in pregnant guinea pig. Am J Physiol. 1988;255:272-9.
38. Dong YL, Yallampalli C. Pregnancy and exogenous steroid treatments modulate the expression of relaxant EP2 and contractile FP receptors in the rat uterus. Biol Reprod. 2000;62:533-9.
Submit your next manuscript to BioMed Central and we will help you at every step:

- We accept pre-submission inquiries

- Our selector tool helps you to find the most relevant journal

- We provide round the clock customer support

- Convenient online submission

- Thorough peer review

- Inclusion in PubMed and all major indexing services

- Maximum visibility for your research

Submit your manuscript at www.biomedcentral.com/submit
(OioMed Central 